\title{
Regulatory RNomics and Gene Expression
}

\author{
Zhumur Ghosh, ${ }^{1}$ Bibekanand Mallick, ${ }^{2}$ Daniel Gautheret, ${ }^{3}$ Pawan Malhotra, ${ }^{4}$ \\ and Ravi Sachidanandam ${ }^{5}$ \\ ${ }^{1}$ Department of Medicine, School of Medicine, Stanford University, Grant Building S140, Stanford, CA 94305, USA \\ ${ }^{2}$ Wadsworth Center, New York State Department of Health, New York, NY 12208, USA \\ ${ }^{3}$ CNRS Institut de Génétique et Microbiologie, Université Paris Sud, Orsay, France \\ ${ }^{4}$ International Centre for Genetic Engineering and Biotechnology, Aruna Asaf Ali Marg, New Delhi 110 067, India \\ ${ }^{5}$ Department of Genetics and Genomic Sciences, Mount Sinai School of Medicine, New York University, NY 10029, USA
}

Correspondence should be addressed to Zhumur Ghosh, ghosh.jhumur@gmail.com

Received 11 October 2009; Accepted 11 October 2009

Copyright (c) 2009 Zhumur Ghosh et al. This is an open access article distributed under the Creative Commons Attribution License, which permits unrestricted use, distribution, and reproduction in any medium, provided the original work is properly cited.

Regulatory RNA research has marked a new paradigm of RNA-directed gene expression regulation and the most promising voice of the post-genomic era in developing new drugs and therapeutics for various diseases. It helps to determine what genes are accessible to each type of cell, a crucial choice for multicellular animals that require, for example, a liver cell to read off one set of genes and a brain cell to be governed by a different set. And it coordinates suites of genes that may be under different control systems but need to act together in response to sudden stresses. The generation of a huge amount of sequences from a number of key organisms and complementary development of computational bio-tools for regulatory RNA annotation and analysis, have accelerated this field of research. The small RNAs have been a boon to scientists bringing reverse genetics to assess the function of a gene. The new regulatory role of RNA began to emerge in the last 10 years as researchers discovered a class of short RNA molecules known as silencing RNAs and a second class called micro-RNAs. And these probably constitute the tip of the iceberg. The opportunity to harness the tantalizing field of regulatory RNA mediated RNA interference (RNAi) pathway in silencing diseasecausing genes hold great promise for the development of therapeutics directed against targets that are not addressable with current medicines. Bioinformatics as well plays a major role and aids in analyzing, interpreting and screening of such RNA sequences and their targets.

MicroRNAs are only the best studied members of the expanding family of regulatory non-coding RNAs that populates all domains of life. MicroRNAs tell us many stories like stories of evolution, with their quickly evolving and adaptable structures that exploded in animal and plant genomes through duplication of existing microRNAs and exaptation of elements that served other functions such as snoRNAs. They tell us stories of development, with their intricate role in the differentiation of so many tissues, including animal brain, immune cells and muscle, as well as plant organs. They are also closely involved in human disease. An astounding 1700 papers have been published about RNA and cancer since the first report of downregulated miRNAs in cancer cells in 2002.

With the revelation that most intergenic and junk DNA in eukaryotic genomes was actually transcribed, a whole new understanding of the non-messenger transcriptome is emerging, where transcripts produced during RNA polymerase initiation or as the result of cleavage by a variety of Dicer-like enzyme are acting in regulating gene expression at many different levels. Regulatory RNAs are also gaining importance in the prokaryotic world in the form of transacting small RNAs and cis-acting riboswitches which, just as their eukaryotic counterparts, are able to switch genes on and off with exquisite accuracy in response to various environmental challenges, triggering deep changes in cellular functions that are reminiscent of the developmental events triggered by miRNAs in multi-cellular organisms. There is a long way ahead in introducing this huge diversity of RNA molecules into our global picture of cell regulatory networks. As our understanding of the functions of small RNAs and 
their mode of regulation continues to increase, we will be able to translate these regulatory RNAs from lab-to-clinic for therapeutic applications.

This area of research has attracted some of the most innovative research groups in the molecular biology and bioinformatics field and as Editors, we are truly privileged that many of these investigators have contributed to this issue. We express our sincere gratitude to the contributing authors as well as to the vision of the Founder Editor, Dr Abdelali Haoudi, for this opportunity provided by the Journal of Biomedicine and Biotechnology.

Zhumur Ghosh

Bibekanand Mallick

Daniel Gautheret

Pawan Malhotra

Ravi Sachidanandam 

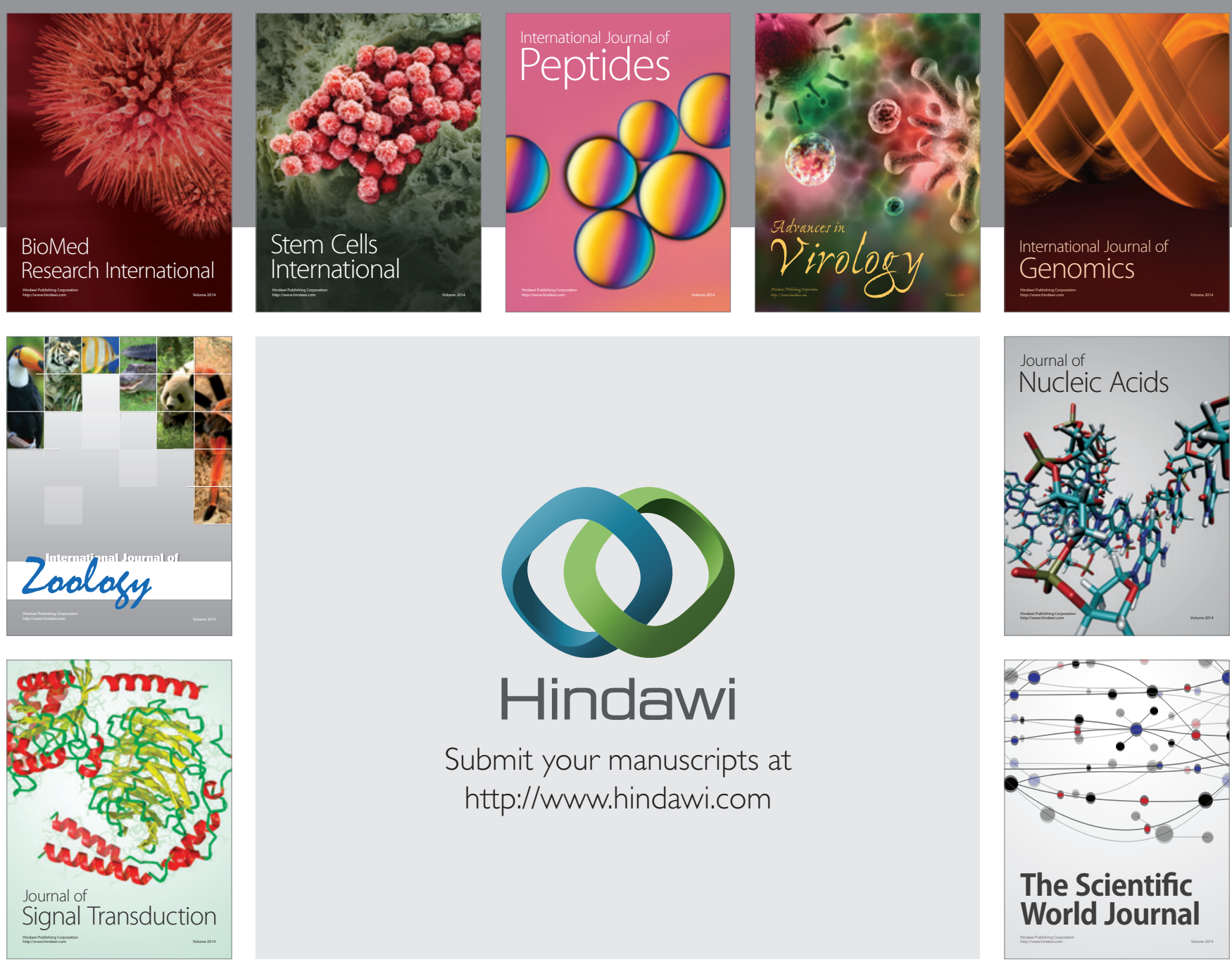

Submit your manuscripts at

http://www.hindawi.com
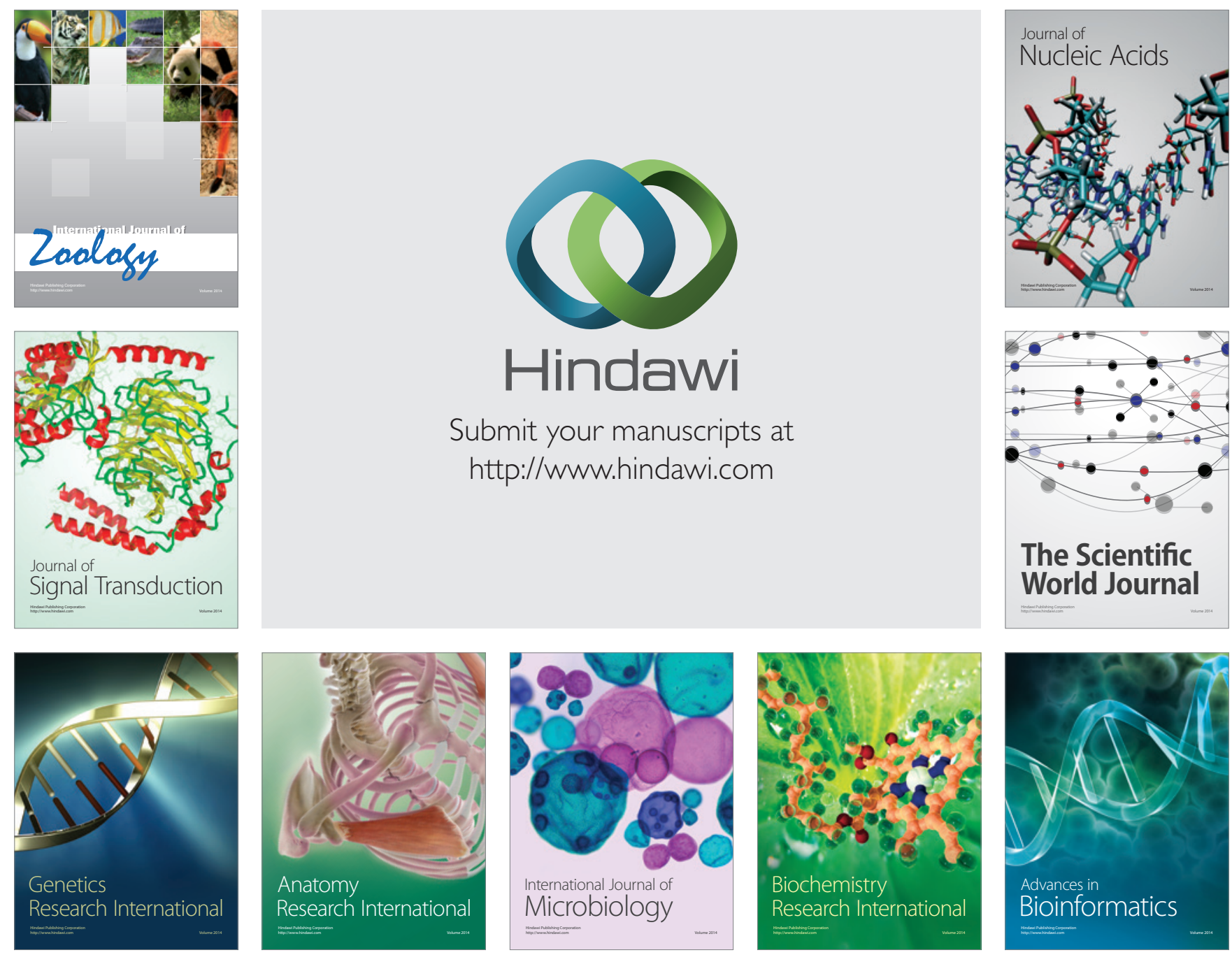

The Scientific World Journal
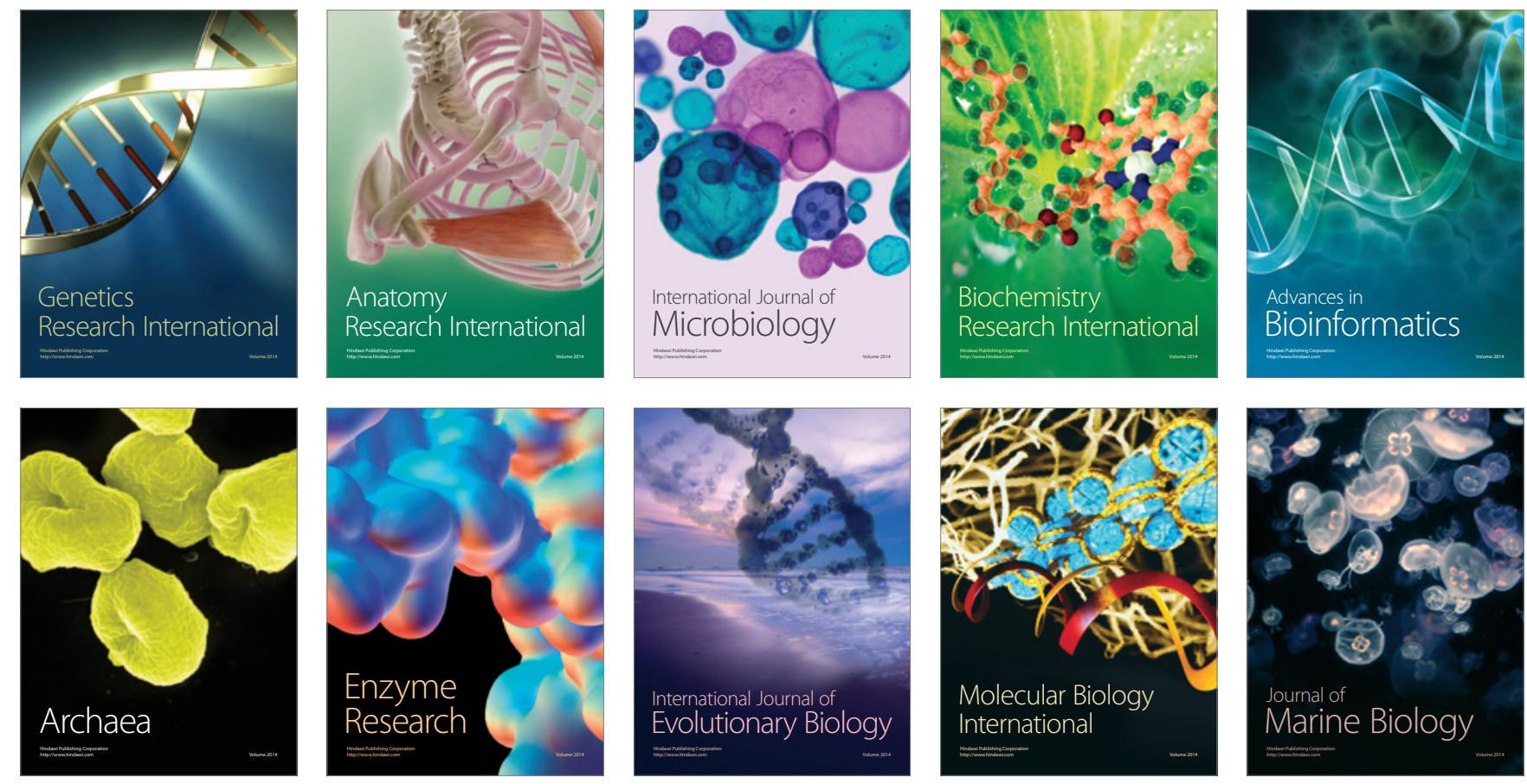